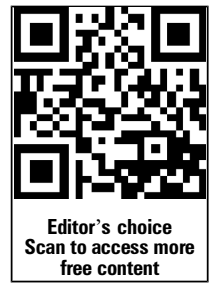

end of article.

\section{Correspondence to} Dr Anita Riipinen, The Social Insurance Institution of Finland, Koskelantie 5 , Helsinki FI-00610, Finland; anita.riipinen@terveystalo.com

AR and MS contributed equally.

Received 30 March 2014 Revised 18 June 2014 Accepted 3 July 2014 Published Online First 29 July 2014
CrossMark

To cite: Riipinen $A$, Sallmén $M$, Hedman $L$, et al. Occup Environ Med 2014;71:836-841.

\title{
Increased risk of human parvovirus B19 infection in day-care employees: a cohort study among pregnant workers during an epidemic in Finland
}

\author{
Anita Riipinen, ${ }^{1,2,3}$ Markku Sallmén, ${ }^{1}$ Lea Hedman, ${ }^{2,4}$ Anneli Ojajärvi, ${ }^{5}$ \\ Marja-Liisa Lindbohm, ${ }^{1}$ Mira Meriluoto, ${ }^{2}$ Heliä-Marja Surcel, ${ }^{6}$ Helena Taskinen, ${ }^{1,7}$ \\ Mika Nuutila, ${ }^{8}$ Riitta Karikoski, ${ }^{9}$ Klaus Hedman, ${ }^{2,4}$ Maria Söderlund-Venermo ${ }^{2}$
}

\begin{abstract}
Background Human parvovirus B19 (B19V) infection during early pregnancy increases the risk of miscarriage. Studies have inconsistently shown an elevated risk of infection among women with occupational contacts with children. Methodological differences, particularly in defining occupational exposure and in the type of reference group, may explain the conflicting findings.

Methods This cohort study compared B19V infections in pregnant day-care employees and healthcare professionals during a B19V epidemic in Finland. Women were identified from the files of nationwide trade unions and the National Supervisory Authority for Welfare and Health. Early-pregnancy maternal B19V IgG was analysed in 3710 women, and infections were defined as seroconversions after analysing in parallel the available umbilical cord blood samples of the 847 seronegative mothers. Independently of the serological status, the actual employment during pregnancy was assessed using registered information on employment history.
\end{abstract}

Results B19V infections were more common among day-care employees $(22 / 331,6.6 \%)$, than among those working in healthcare (12/326, 3.7\%). The adjusted HRs of B19V infection, using proportional hazard regression, was $2.63(95 \% \mathrm{Cl} 1.27$ to 5.46$)$ among all women and $5.59(95 \% \mathrm{Cl} 1.40$ to 22.4$)$ among nulliparous women. Conclusions Day-care employees are at an increased risk of B19V infection, which warrants preventive measures.

\section{INTRODUCTION}

Human parvovirus B19 (B19V) was first described in $1975 .{ }^{1} \mathrm{~B} 19 \mathrm{~V}$ infection causes erythema infectiosum, a common rash in preschool or school-aged children, arthropathies including arthritis in adults, transient aplastic crisis in patients with an underlying haematological disease, chronic bone marrow failure in immunocompromised patients and some other manifestations of diseases. ${ }^{2-4} \mathrm{~B} 19 \mathrm{~V}$ is transmitted not only via respiratory secretions but also vertically from mother to fetus at a transmission rate of $25-50 \% .^{5}{ }^{6}$ Antenatal B19V infection can lead to hydrops fetalis, miscarriage (fetal loss before week 22 of gestation) and intrauterine fetal death (at or after week 22). ${ }^{7}$ The excess risk of fetal loss (6-9\%) seems to be restricted to maternal

\section{What this paper adds}

- Studies have inconsistently shown an elevated risk of human parvovirus B19 (B19V) infection among women with occupational contact with children. High-quality studies have indicated an increased risk of miscarriage and intrauterine fetal death among the infected women.

- We compared infections in pregnant day-care workers and healthcare professionals with a similar socioeconomic status but without occupational contact with children.

- We observed an increased risk of B19V infection among day-care employees. The difference in the infection risk was even more pronounced in the analysis of nulliparous women, eliminating the effect of the women's own children.

- Based on our findings and studies of adverse pregnancy outcomes, we propose the following guideline: during a B19V outbreak in a day-care unit or elementary school, pregnant B19V-seronegative employees should be transferred from the epidemic workplace to another workplace or allowed a leave of absence for approximately 2 months.

infection at the end of the first or the beginning of the second trimester. 589

B19V outbreaks occur mostly in the spring, with major epidemics every few years. In one large study, the annual incidence of $\mathrm{B} 19 \mathrm{~V}-\mathrm{IgG}$ seroconversion during pregnancy was $1.5 \%$ in a non-epidemic period and $13 \%$ in an epidemic period. ${ }^{10}$

In general, most infections of pregnant women are transmitted by the women's own children. ${ }^{10}$ The occupational risk of B19V infection has been reported as being elevated among day-care employees and school teachers in some ${ }^{10-13}$ but not all studies. ${ }^{14-16}$ Nevertheless, the routine exclusion of pregnant day-care employees from high-risk workplaces is not generally recommended. ${ }^{5} 1013$ 17-19

We investigated whether day-care employees are at increased risk of $\mathrm{B} 19 \mathrm{~V}$ infection. B19V infections were defined as B19V-IgG seroconversions between early pregnancy and birth. Unlike previous 
studies, we restricted the comparison to employed women and used women in healthcare as a comparison group. We also examined in detail the impact of (1) work in general, (2) work with children and (3) the participants' own children, in the routes of infection. The objective of this study was to obtain a more thorough scientific basis than previously for the assessment of the occupational safety of pregnant women at risk of B19V infection.

\section{METHODS}

Study population, samples and data sources

A cohort study of B19V infection was conducted among pregnant day-care and healthcare employees in Finland. We identified 60926 employees born in 1946 or later, and the final study population consisted of 847 susceptible women who were pregnant during the period September 1992-August 1993. The study period includes a major B19V epidemic.

The study group consisted of professional children's nurses and nursery school teachers (figure 1), whereas the comparison group included healthcare professionals with similar socioeconomic status, but with little or no occupational contact with children. The latter occupations were physiotherapists, dispensers, opticians, masseuses, rehabilitation nurses, dental assistants and dental hygienists. The nursery school teachers were identified from the files of the Trade Union of Education and the Union of Professional Social Workers. The women who qualified in the other occupations were identified in the register of the National Supervisory Authority for Welfare and Health. The employee data included personal identity number (ID), occupation, and date of qualification or union membership. We identified the women from the different registers using their IDs.

\section{Data on early-pregnancy and umbilical cord serum samples}

To identify the pregnant women of interest to the present study, we linked the employee data with two data sets of serum samples, the first ones taken in early pregnancy and the second at birth (figure 1). In Finland, maternal early-pregnancy sera are routinely collected and stored by the Finnish Maternity Cohort (FMC) maintained by the National Institute for Health and Welfare. The data include ID, the sampling date and the expected date of confinement (EDC). In addition, during the period September 1992-August 1993, cord blood samples

Workers qualified in day-care and health care occupations

The Trade Union of Education and the Union of Professional Social Workers:

- Nursery school teachers

The Register of the National Authority for Medicolegal Affairs:

- Children's nurses, physiotherapists, dispensers, opticians, masseuses, practical rehabilitation nurses, and dental nurses/hygienists

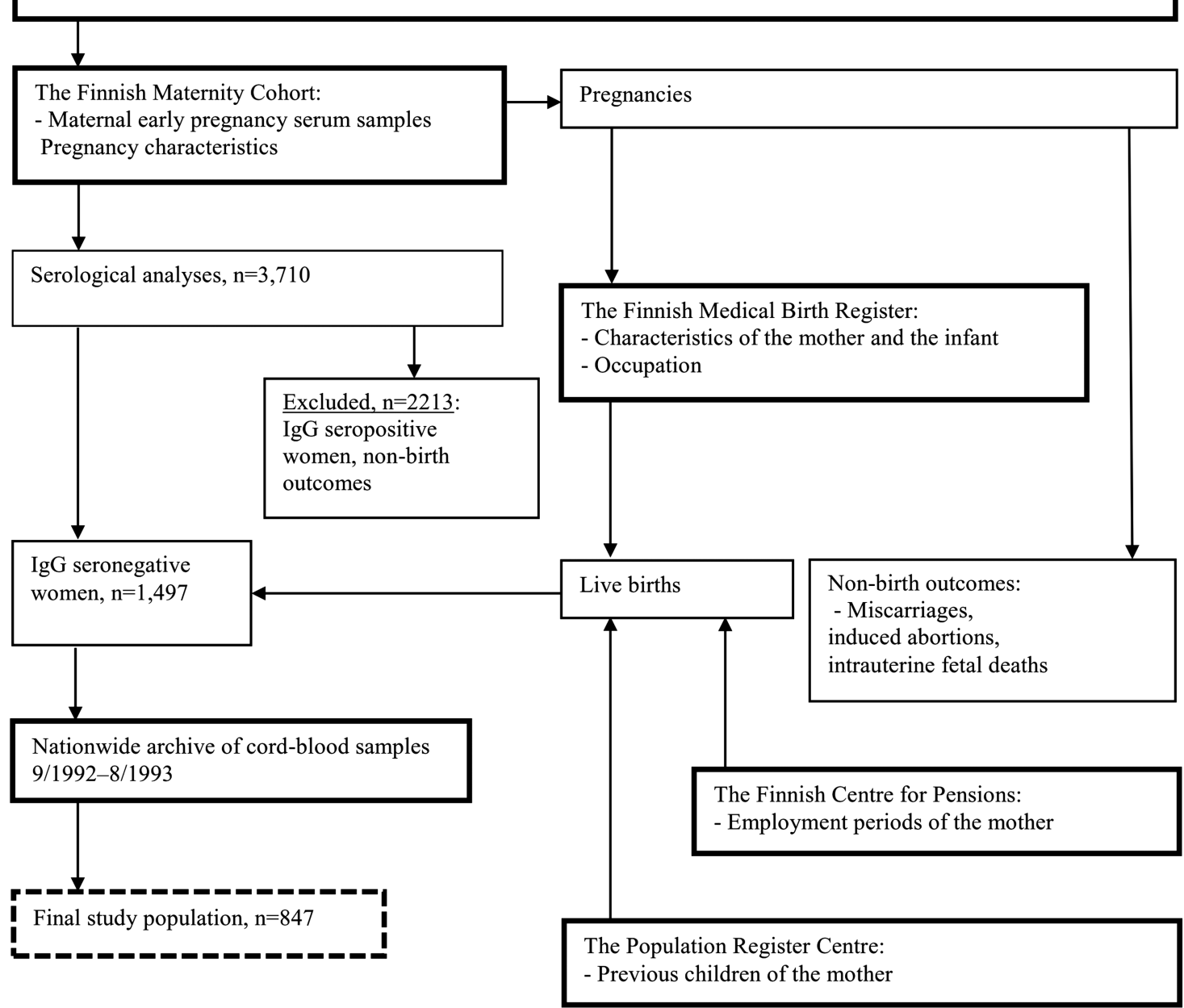

Figure 1 Design of a cohort study of human parvovirus B19 infections among day-care and healthcare employees, Finland, $1992-1993$. 
$(\mathrm{N}=49680)$ were drawn at term for the screening of congenital hypothyreosis by the National Institute for Health and Welfare, with a nationwide coverage of $\sim 76 \%$ (the number of annual births was about 65000 in 1992 and 1993).

We included each woman's first pregnancy that could have resulted in childbirth during the period of the archived umbilical cord serum sampling, that is, September 1992-August 1993 $(n=4276)$. The early-pregnancy sera of 132 women were not found. We further restricted the study to women who had graduated or joined the trade union prior to the serum sampling date $(n=3710)$. A total of 3430 pregnancies $(92.5 \%)$ resulted in live birth and $13(0.4 \%)$ in intrauterine fetal death. The 267 $(7.2 \%)$ non-birth outcomes consisted of miscarriages and induced abortions. For births and intrauterine fetal deaths, we obtained information from the Finnish Medical Birth Register (MBR) on date of birth, gestation length, hospital district and previous births. The MBR was established in 1987 and contains data on all newborn infants and on all mothers who have given birth in Finland. ${ }^{20}$

All the eligible early-pregnancy serum samples $(n=3710)$ were examined for B19V IgG by an indirect enzyme immunoassay using streptavidin-bound biotinylated virus-like particles of virus protein 2 as an antigen. ${ }^{21}$ The cut-off absorbance for a positive IgG result was 0.198 . IgG-seronegative women with live births $(n=1497)$ were eligible for our study of incident B19V infections. For this purpose, the available cord blood samples $(n=847)$ in parallel with retesting of each corresponding early-pregnancy sample were examined for IgG. The cord blood samples of 650 women (43\%) were not studied, because: (1) the hospital did not deliver cord blood samples $(n=523)$; (2) the sample was inaccessible $(n=127)$.

\section{Time windows for B19V infections and employment}

Based on the known B19V IgG appearance kinetics, ${ }^{22}$ the follow-up for seroconversion events began 14 days before the serum sampling date. Likewise, the end of the follow-up was set at 14 days before birth (ie, cord blood sampling). The mean length of the follow-up was 205 days (range 90-493); the longer follow-up periods are due to 18 women whose earlypregnancy sample was obtained during a prior pregnancy. In Finland, maternity leave begins no later than 35 days prior to the EDC. Therefore, we assessed employment for a period starting at the beginning of follow-up but ceasing either 5 weeks prior to EDC, or 2 weeks before delivery, whichever came first.

\section{Defining occupation and employment status during}

seroconversion follow-up

We collected information from various registers to specify the prenatal occupation and employment status of the women during the seroconversion follow-up (figure 1). First, all the 847 women were classified into either day-care $(n=432)$ or healthcare $(\mathrm{n}=415)$ occupations by education. Second, we defined occupation during follow-up using the MBR information resulting in 384 women in day care and 369 women in healthcare. Finally, to define employment-verified occupation, we checked prenatal employment using the registers of the Finnish Centre for Pensions, in which all salaried employments are registered. This final seroconversion population of 847 women with employment-verified occupation comprised 331 day-care and 326 healthcare employees with registered employment, and in addition, 60 women with other occupations, and 130 unemployed women.

\section{Statistical analysis}

HRs of prenatal B19V infection between occupational groups were assessed with proportional hazard regression using the SAS PHREG procedure (SAS V.9.2, SAS Institute Inc, Cary, North Carolina, USA). The time-dependent interaction between occupation groups and the follow-up period was statistically nonsignificant, confirming that the proportional hazards assumption was justified. ${ }^{23}$

\section{Factors considered in the analyses}

Working practices may change with increasing job seniority. Therefore, we created a 'job seniority' variable as years from graduation/joining the trade union, with three groups: 0-4, 5-9 and $\geq 10$ years. We also recognised spatiotemporal variability in occupational groups and infections. The women from the Capital (Helsinki) Region were more likely to work in day care but less likely to experience B19V infection than the women in other parts of Finland. We therefore defined a variable Capital Region. The occurrence of seroconversions was higher for late follow-up periods, early-pregnancy sample in or after September 1992 and/or a birth in or after May 1993. We defined the corresponding follow-up periods as having occurred during a high-risk period. Other periods were thus defined as low-risk periods of B19V infection.

We selected variables into the multivariable models as follows. Variables for women's own living children, assessed using information from the MBR and from the Population Information System maintained by the Population Register Centre, were forced into the models. Both the number of children and their ages were considered. From among other variables, we selected those that were associated with the risk of infection $(p<0.05)$, or had an impact on the relative risk of infection between day-care and healthcare employees.

\section{Sensitivity analyses}

We used the employment-verified occupation as the main occupational classification in assessing the impact of day-care work on the occurrence of B19V infection. To learn from the impact of exposure misclassification, we studied the occurrence of B19V infection by also using other definitions of occupation with varying potential for occupational misclassification. Moreover, an analysis restricted to nulliparous women was performed to eliminate the effect of the women's own children. We also recognised an additional source of exposure misclassification in women with young children. In Finland, women with children $<3$ years of age are entitled to childcare leave. Therefore, we conducted an analysis excluding women who were employed at the beginning of the study pregnancy and had a $<3$-year-old child.

The study was double blinded: the occupation and employment of the women, and the serological classifications were assessed independently of each other. Permission for use of the MBR was obtained from the Ministry of Social Affairs and Health. Permission for use of the registers of the FMC, the Finnish Centre for Pensions, Statistics Finland, the Trade Union of Education and the Union of Professional Social Workers was obtained from the organisations themselves.

\section{RESULTS}

The women with day-care education were more likely to be B19V-IgG seropositive (59\%) than the women with healthcare education $(55 \%$; table 1$)$. Seropositivity increased in relation to the number of the women's own living children but the increase in relation to the women's age and job seniority was weaker. 
Table 1 Characteristics of women and human parvovirus B19 (B19V) IgG seroprevalence at the time of the first antenatal visit in a cohort study among women with day-care and healthcare education, Finland, 1992-1993

\begin{tabular}{|c|c|c|c|c|c|c|c|c|}
\hline \multirow[b]{3}{*}{ Characteristics } & \multicolumn{4}{|c|}{ Day-care education } & \multicolumn{4}{|c|}{ Healthcare education } \\
\hline & \multicolumn{2}{|c|}{ Women } & \multicolumn{2}{|c|}{ B19V seroprevalence } & \multicolumn{2}{|c|}{ Women } & \multicolumn{2}{|c|}{ B19V seroprevalence } \\
\hline & $\mathbf{N}$ & Per cent & $\mathrm{N}$ & Per cent & $\mathbf{N}$ & Per cent & $\mathbf{N}$ & Per cent \\
\hline Total & 1966 & & 1162 & 59.1 & 1744 & & 957 & 54.9 \\
\hline \multicolumn{9}{|l|}{ Age, years } \\
\hline 19-24 & 212 & 10.8 & 120 & 56.6 & 209 & 12.0 & 109 & 52.2 \\
\hline $25-29$ & 935 & 47.6 & 548 & 58.6 & 700 & 40.1 & 383 & 54.7 \\
\hline $30-34$ & 638 & 32.4 & 383 & 60.0 & 582 & 33.4 & 326 & 56.0 \\
\hline $35-48$ & 181 & 9.2 & 111 & 61.3 & 253 & 14.5 & 139 & 54.9 \\
\hline \multicolumn{9}{|c|}{ Number of living children } \\
\hline 0 & 735 & 37.4 & 423 & 57.6 & 738 & 42.3 & 384 & 52.0 \\
\hline 1 & 708 & 36.0 & 413 & 58.3 & 589 & 33.8 & 330 & 56.0 \\
\hline 2 & 370 & 18.8 & 227 & 61.4 & 286 & 16.4 & 162 & 56.6 \\
\hline$\geq 3$ & 153 & 7.8 & 99 & 64.7 & 131 & 7.5 & 81 & 61.8 \\
\hline \multicolumn{9}{|c|}{ Job seniority, years } \\
\hline $0-4$ & 666 & 33.9 & 389 & 58.4 & 579 & 33 & 323 & 55.8 \\
\hline $5-9$ & 900 & 44.8 & 527 & 58.6 & 687 & 39 & 370 & 53.9 \\
\hline$\geq 10$ & 400 & 20.4 & 246 & 61.5 & 478 & 27 & 264 & 55.2 \\
\hline
\end{tabular}

The day-care employees were more likely to be infected during pregnancy $(6.6 \% ; 22 / 331)$ than those in healthcare $(3.7 \% ; 12 / 326)$, corresponding to annual seroconversion rates of $12.2 \%$ and $6.7 \%$, respectively (table 2 ). The women in the Capital Region were at a lower risk of $\mathrm{B} 19 \mathrm{~V}$ infection than the women in other parts of Finland (adjusted HR $(\mathrm{aHR})=0.57$, $95 \%$ CI 0.26 to 1.27 ). As expected, the risk of infection increased with the number of children of the woman.

The aHR of B19V infection for day-care work was 2.63 (95\% CI 1.27 to 5.46 ; table 2). HRs for day-care work according to the various definitions of occupation as well as that among nulliparous women are presented in figure 2. The strongest association can be seen among nulliparous women, though with a wide CI $(\mathrm{aHR}=5.59$, 95\% CI 1.40 to 22.4). HRs were higher in the analysis using employment-verified occupation than in analyses using education or self-reported occupation as a measure of exposure. The relative risk for day-care work was also higher when the women entitled to childcare leave were excluded from the model. This restriction is supposedly least prone to occupational misclassification.

We explored the impact of work and the women's own living children on the risk of $\mathrm{B} 19 \mathrm{~V}$ infection in more detail. The risk increased along with the number of the women's own children in the entire data (aHR for continuous variable is $1.44,95 \% \mathrm{CI}$ 1.12 to 1.83$)$ and among all the employed women $(\mathrm{aHR}=1.70$, $95 \%$ CI 1.29 to 2.24). However, this tendency was not seen in the small group of non-employed women $(\mathrm{N}=130, \mathrm{aHR}=0.86$, $95 \%$ CI 0.41 to 1.81 ).

\section{DISCUSSION}

We found a 2.6-fold increased risk of B19V infection among day-care employees compared with women employed in healthcare. The association was stronger among nulliparous women. In addition, the slightly elevated seroprevalence among day-care employees points to an elevated cumulative occupational risk.

In a population-based Danish study, ${ }^{10}$ women with day-care education were at a three-time higher risk of B19V infection compared with other women. Another Danish study found a higher risk of infection among employed than among non-employed women. ${ }^{24}$ However, the risk for those working with children did not deviate from that of other working women. Additionally, an elevated risk in work with children has been reported in a number of other studies, ${ }^{11-13}$ although not in all studies. ${ }^{14-16}$ We focused on employed women and, unlike the other studies, ${ }^{10} 24$ we used women with a similar socioeconomic status as a comparison group. This study decision may have made the compared groups more similar also in precautions such as hand washing when taking care of their own children. Other methodological differences could also have affected the contradictory results.

A major strength of our study was the large size of the occupational cohort and the fact that the study took place during a major B19V epidemic. Occupational exposure and the employment status of all participants were specified using several nationwide registers. Many known or potential risk factors for $\mathrm{B} 19 \mathrm{~V}$ infection, such as age and the number of the mother's own living children, the age of the mother, area and period were considered in the analyses.

\section{Misclassification of occupation or employment}

Education was used as a measure of women's occupational exposure in a major Danish population-based study, and researchers reasoned that misclassification of occupational exposure would have been limited. ${ }^{10}$ In line with their reasoning, we found similar results in the analyses using education or self-reported occupation as an indicator of occupational exposure. However, we found a higher relative risk of B19V infection for day-care work when we used employment-verified occupation as an exposure measure instead of more crude exposure indicators. The observed risk further increased substantially after excluding mothers potentially on childcare leave, affecting about $20 \%$ of the mothers in day care, and $15 \%$ of the mothers in healthcare. These findings point to the importance of the quality of exposure assessment and to the need to verify employment during pregnancy when assessing occupational risks. 
Table 2 HR of human parvovirus B19 (B19V) seroconversions among the day-care employees compared with the women in healthcare $(\mathrm{N}=847)$, Finland, 1992-1993

\begin{tabular}{|c|c|c|c|c|c|c|c|}
\hline \multirow[b]{2}{*}{ Characteristics } & \multirow{2}{*}{$\begin{array}{l}\text { Women } \\
\mathrm{N}\end{array}$} & \multicolumn{2}{|c|}{ Seroconversions } & \multicolumn{2}{|c|}{ Unadjusted } & \multicolumn{2}{|c|}{ Adjusted } \\
\hline & & $\mathbf{N}$ & Per cent & \multicolumn{2}{|c|}{$\mathrm{HR}(95 \% \mathrm{Cl})^{*}$} & \multicolumn{2}{|c|}{ HR $(95 \% \mathrm{Cl}) \dagger$} \\
\hline \multicolumn{8}{|l|}{ Employment } \\
\hline No & 130 & 6 & 4.6 & 1.00 & (Reference) & 1.00 & (Reference) \\
\hline Yes & 717 & 36 & 5.0 & 1.10 & (0.46 to 1.60$)$ & 1.22 & (0.43 to 3.42$)$ \\
\hline \multicolumn{8}{|c|}{ Employment-verified occupation } \\
\hline Healthcare worker & 326 & 12 & 3.7 & 1.00 & (Reference) & 1.00 & (Reference) \\
\hline Day-care worker & 331 & 22 & 6.6 & 2.13 & (1.05 to 4.32$)$ & 2.63 & (1.27 to 5.46$)$ \\
\hline Other occupation & 60 & 2 & 3.3 & 0.93 & (0.21 to 4.17$)$ & 0.85 & (0.18 to 4.09$)$ \\
\hline \multicolumn{8}{|l|}{ Age, years } \\
\hline 19-24 & 110 & 8 & 7.3 & 1.00 & (Reference) & 1.00 & (Reference) \\
\hline $25-29$ & 373 & 14 & 3.8 & 0.63 & (0.26 to 1.50$)$ & 0.48 & (0.18 to 4.09$)$ \\
\hline $30-34$ & 265 & 13 & 4.9 & 0.88 & (0.37 to 2.14$)$ & 0.53 & (0.18 to 1.53$)$ \\
\hline $35-48$ & 99 & 7 & 7.1 & 1.44 & $(0.52$ to 4.00$)$ & 0.83 & (0.24 to 2.85$)$ \\
\hline \multicolumn{8}{|c|}{ Number of living children } \\
\hline 0 & 369 & 14 & 3.8 & 1.00 & (Reference) & 1.00 & (Reference) \\
\hline 1 & 283 & 13 & 4.6 & 1.29 & (0.60 to 2.75$)$ & 1.38 & (0.60 to 3.18$)$ \\
\hline 2 & 137 & 8 & 5.8 & 1.62 & (0.67 to 3.89$)$ & 1.90 & (0.68 to 5.34$)$ \\
\hline$\geq 3$ & 58 & 7 & 12.1 & 4.81 & (1.92 to 12.0$)$ & 6.27 & (2.04 to 19.2 ) \\
\hline \multicolumn{8}{|c|}{ High infection risk period } \\
\hline No & 473 & 13 & 2.8 & 1.00 & (Reference) & 1.00 & (Reference) \\
\hline Yes & 374 & 29 & 7.8 & 3.53 & (1.83 to 6.81 ) & 3.91 & (2.01 to 7.59$)$ \\
\hline \multicolumn{8}{|l|}{ Capital Region } \\
\hline No & 552 & 34 & 6.1 & 1.00 & (Reference) & 1.00 & (Reference) \\
\hline Yes & 285 & 8 & 2.8 & 0.52 & (0.24 to 1.13 ) & 0.57 & (0.26 to 1.27$)$ \\
\hline \multicolumn{8}{|l|}{ Job seniority, years } \\
\hline $0-4$ & 301 & 14 & 4.6 & 1.00 & (Reference) & & \\
\hline $5-9$ & 344 & 17 & 4.9 & 1.10 & (0.54 to 2.23$)$ & & \\
\hline$\geq 10$ & 202 & 11 & 5.5 & 1.43 & (0.64 to 3.16$)$ & & \\
\hline
\end{tabular}

${ }^{*}$ Seven univariable models; the model for employment-verified occupation also includes employment. tMultivariable model including all variables except job seniority.

The present register-based study might still suffer from a minor residual misclassification of occupation. Based on our earlier comparison of day-care and healthcare occupations between MBR and Statistics Finland, ${ }^{25}$ we assume that employment-verified occupation may still be misclassified in about $10 \%$ of the studied women. Residual misclassification of occupation may have caused a marginal attenuation of the true effect.

\section{Impact of work and children on infection risk}

We found approximately 10 excess infections in day care. Given that these infections are caused by work, they supposedly are confined to actual working hours. In Finland (about 200 working days per year á 7-8 h), working hours constitute less than $20 \%$ of the total time. It can therefore be reasoned that, during actual working hours, the risk of B19V infection could in reality have been as much as 10-15 times higher in day care than in healthcare.

Because of the major impact of the women's own children on B19V infections, ${ }^{10}$ studies including parous women tend to underestimate the relative risk of work in day care compared with that in other work. We explored the associations of the B19V infection with the number of the women's own children and employment in more detail, and recognised the highest relative risk for day-care work among nulliparous women. In addition, the risk of infection increased along with a higher number

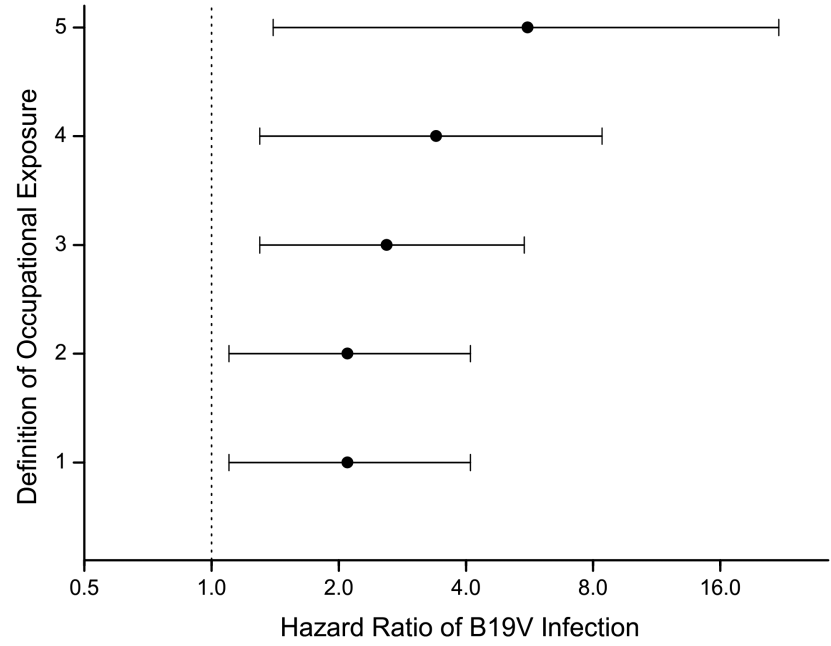

Figure 2 Sensitivity analyses of the risk of human parvovirus B19 (B19V) infection among day-care employees using different exposure definitions. The figures show adjusted HRs of infection and $95 \% \mathrm{Cls}$. The definitions of occupational exposure are: (1) education (432 day-care employees and 415 healthcare employees), (2) occupation ( $n=384$ and 369), (3) occupation with verified employment ( $n=331$ and 326), (4) as in definition 3, but women entitled to childcare leave excluded ( $n=245$ and 265$),(5)$ as in definition 4, but only nulliparous women included ( $n=144$ and 158). 
of children among employed women, but not among non-employed women. Based on these findings, we hypothesise that day-care-aged children of employed women may have more contacts with age mates than children of non-employed women, and thus have a higher risk of being infected and of transmitting the infection to their mothers. Analyses among nulliparous women are more appropriate in assessing the genuine impact of work and work with children.

\section{Loss of follow-up}

Obviously, no umbilical cord blood samples were available from the succumbed fetuses $(n=94)$. These comprise intrauterine fetal deaths $(n=6)$, miscarriages and induced abortions. As B19V is not considered a teratogen, we did not expect the numbers of induced abortions to have risen above baseline. However, because B19V infection during the first half of pregnancy increases the risk of miscarriage, ${ }^{5} 8920$ our numbers of seroconversions, based on cord blood, slightly underestimated the absolute risk of $\mathrm{B} 19 \mathrm{~V}$ infection. Nevertheless, the occupational comparisons of infections may not be influenced because the risk of fetal death of the affected fetus is unlikely to depend on the mother's occupation. Altogether 650 (43\%) cord blood samples were missing due to non-participation of hospitals or storage depletion. These losses increase the CIs but are supposedly unrelated to occupational B19V infections.

Our findings form evidence that day-care employees are at an increased risk of $\mathrm{B} 19 \mathrm{~V}$ infection, and this should be taken into account in the assessment of occupational safety measures for pregnant women. During a B19V outbreak in a day-care unit or elementary school, B19V-seronegative pregnant employees should be transferred from the epidemic workplace to another workplace. Maternity leave is another preventive procedure. At a particular affected day care or school the duration of a B19V outbreak, including a safety period, is approximately 2 months, during which time such an allowed leave of absence is needed if a change in working place is not possible.

Because the risk of infection increases in proportion to the number of children, studies including parous women tend to underestimate the impact of day-care work as a risk factor. An analysis among nulliparous women provides more valid results. In future studies, not only the number and ages of the women's own children, but also the number of children's contacts with their age mates should be considered as a transmitter of B19V infection in countries with universal access to day care.

\footnotetext{
Author affiliations

${ }^{1}$ Centre of Expertise for Health and Work Ability, Finnish Institute of Occupational Health, Helsinki, Finland

${ }^{2}$ Department of Virology, Haartman Institute, University of Helsinki, Helsinki, Finland ${ }^{3}$ The Social Insurance Institution of Finland, Insurance Region for Southern Finland, Helsinki, Finland

${ }^{4}$ Laboratory Services, HUSLAB, Department of Virology and Immunology, Helsinki University Central Hospital, Hospital District of Helsinki and Uusimaa, Helsinki, Finland

${ }^{5}$ Finnish Institute of Occupational Health, Centre of Creating Solutions, Helsinki, Finland

${ }^{6}$ National Institute for Health and Welfare, Oulu, Finland

7 University of Helsinki, Hjelt Institute, Helsinki, Finland

${ }^{8}$ Department of Obstetrics and Gynecology, Helsinki University Central Hospital, Helsinki, Finland

${ }^{9}$ Department of Pathology, Helsinki University Central Hospital, HUSLAB, Helsinki, Finland
}

Contributors HT, AR, KH, MS-V, RK, M-LL, MS and MN conceived the study. MS-V, AR and KH designed the serological analyses. H-MS provided the samples. AR, MM and LH studied the samples, supervised by MS-V and KH. MS, with the group, designed the epidemiological study and prepared the epidemiological data. MS combined it with the serological data. AO and MS conducted the statistical analyses (with group). All authors contributed to the interpretation of the results. AR and MS drafted the manuscript. MS-V, KH, M-LL and HT revised the manuscript critically for important intellectual content. All authors reviewed the final version of the manuscript and approved it to be published.

Funding This work was supported by The Academy of Finland (109905, 1122539), the Sigrid Jusélius Foundation, the Research Funds of the University of Helsinki, the Helsinki University Central Hospital Research \& Education and Research \& Development funds and the Medical Society of Finland.

Competing interests None.

Ethics approval The study was approved by the Coordinating Ethics Committee of the Helsinki and Uusimaa Hospital District.

Provenance and peer review Not commissioned; externally peer reviewed.

\section{REFERENCES}

1 Cossart YE, Field AM, Cant B, et al. Parvovirus like particles in human sera. Lancet 1975:1:72-3.

2 Pattison JR, Jones SE, Hodgson J, et al. Parvovirus infections and hypoplastic crisis in sickle-cell anaemia. Lancet 1981;1:664-5.

3 Reid DM, Brown T, Reid TMS, et al. Human parvovirus-associated arthritis: a clinical and laboratory description. Lancet 1985;1:422-5.

4 Kurtzman GJ, Cohen B, Meyers P, et al. Persistent B19 parvovirus infection as a cause of severe chronic anaemia in children with acute lymphocytic leukaemia. Lancet 1988;2:1159-62.

5 Public Health Laboratory Service Working Party on Fifth Disease. Prospective study of human parvovirus (B19) infection in pregnancy. BMJ 1990;300:1166-70.

6 Koch WC, Harger JH, Barnstein B, et al. Serologic and virologic evidence for frequent intrauterine transmission of human parvovirus B19 with a primary maternal infection during pregnancy. Pediatr Infect Dis J 1998;17:489-94.

7 Brown $T$, Anand A, Ritchie LD, et al. Intrauterine parvovirus infection associated with hydrops fetalis. Lancet 1984;2:1033-4.

8 Miller E, Fairley CK, Cohen BJ, et al. Immediate and long term outcome of human parvovirus B19 infection in pregnancy. Br J Obstet Gynaecol 1998;105:174-8.

9 Enders $\mathrm{M}$, Weidner $\mathrm{A}$, Zoellner I, et al. Fetal morbidity and mortality after acute human parvovirus B19 infection in pregnancy: prospective evaluation of 1018 cases. Prenat Diagn 2004;24:513-18.

10 Valeur-Jensen AK, Pedersen CB, Westergaard T, et al. Risk factors for parvovirus B19 infection in pregnancy. JAMA 1999;281:1099-105.

11 Gillespie SM, Cartter ML, Asch S, et al. Occupational risk of human parvovirus B19 infection for school and day-care personnel during an outbreak of erythema infectiosum. JAMA 1990;263:2061-5.

12 Adler SP, Manganello AM, Koch WC, et al. Risk of human parvovirus B19 infections among school and hospital employees during endemic periods. J Infect Dis 1993; 168:361-8

13 Cartter ML, Farley TA, Rosengren S, et al. Occupational risk factors for infection with parvovirus B19 among pregnant women. J Infect Dis 1991;163:282-5.

14 Harger JH, Adler SP, Koch WC, et al. Prospective evaluation of 618 pregnant women exposed to parvovirus B19: risks and symptoms. Obstet Gynecol 1998;91:413-20.

15 Koch WC, Adler SP. Human parvovirus B19 infections in women of childbearing age and within families. Pediatr Infect Dis J 1989;8:83-7.

16 Stelma FF, Smismans A, Goossens VJ, et al. Occupational risk of human cytomegalovirus and parvovirus B19 in female day care personnel in the Netherlands; a study based on seroprevalence. Eur J Clin Microbiol Infect Dis 2009;28:393-7.

17 Parvovirus, erythema infectiosum, and pregnancy. Committee on infectious diseases, 1989-1990. Pediatrics 1990;85:131-3.

18 Crowcroft NS, Roth CE, Cohen BJ, et al. Guidance for control of parvovirus B19 infection in healthcare settings and the community. J Public Health Med 1999;21:439-46.

19 Lassen J, Jensen AK, Bager $P$, et al. Parvovirus B19 infection in the first trimester of pregnancy and risk of fetal loss: a population-based case-control study. Am J Epidemiol 2012;176:803-7.

20 Gissler $\mathrm{M}$, Teperi J, Hemminki $\mathrm{E}$, et al. Data quality after restructuring a national medical registry. Scand J Soc Med 1995;23:75-80.

21 Kaikkonen L, Lankinen H, Harjunpää I, et al. Acute-phase-specific heptapeptide epitope for diagnosis of parvovirus B19 infection. J Clin Microbiol 1999;37:3952-6.

22 Anderson MJ, Higgins PG, Davis LR, et al. Experimental parvoviral infection in humans. J Infect Dis 1985;152:257-65.

23 Allison PD. Survival analysis using the SAS system: a practical guide. Cary, NC: SAS Institute Inc., 1995.

24 Jensen IP, Thorsen $\mathrm{P}$, Jeune $\mathrm{B}$, et al. An epidemic of parvovirus B19 in a population of 3,596 pregnant women: a study of sociodemographic and medical risk factors. BJOG 2000;107:637-43.

25 Riipinen $A$, Sallmén $M$, Taskinen $H$, et al. Pregnancy outcomes among day-care employees in Finland. Scand J Work Environ Health 2010;36:222-30. 